\title{
Cultura Audiovisual en la Formación Profesional del Sistema Educativo
}

\section{Audiovisual Culture Within the Education System's Vocational Education and Training}

Luis García Domínguez

\section{El sistema de FP en España}

Hacer una fotografía nítida de la Formación Profesional en España es tan complejo como lo es el propio sistema. Desde la aprobación de la Ley Orgánica 1/1990, de 3 de octubre, de Ordenación General del Sistema Educativo, LOGSE, y, posteriormente, con la Ley orgánica 5/2002, de 19 de junio, de las Cualificaciones y de la Formación Profesional, la FP está organizada en dos subsistemas: la Formación Profesional del Sistema Educativo y la Formación Profesional para el Empleo. A este sistema común podríamos añadir las enseñanzas profesionales de régimen especial de enseñanzas artísticas, que están reguladas como si fuesen títulos de Formación Profesional también desde la mencionada LOGSE.

Para la FP del Sistema Educativo y para la FP para el Empleo, el marco de referencia es el Catálogo Nacional de Cualificaciones Profesionales (SNCFP, 2020), que le da coherencia a los dos subsistemas, pues es la fuente común donde se alimentan el diseño curricular de los títulos de FP del sistema educativo y los Certificados de Profesionalidad del sistema para el empleo.

El Instituto Nacional de Cualificaciones Profesionales, organismo compuesto por especialistas del Ministerio de Trabajo y del Ministerio de Educación y FP, es el encargado de detectar, describir, definir y publicar oficialmente las cualificaciones necesarias para acreditar los perfiles profesionales de nuestra estructura productiva. Para ello, el Instituto Nacional de Cualificaciones Profesionales, bien de oficio o bien a instancia de un grupo de profesionales, de un sindicato, de la patronal empresarial o gremial o de cualquier asociación civil, va añadiendo, modificando 0 eliminando estas cualificaciones profesionales, que serán la base a partir de la que organizarán los títulos y certificados.

Pero ¿qué es una cualificación profesional? Tal como indica el propio Ministerio de Educación y Formación Profesional en su sitio web específico para la FP, todofp.es, una cualificación se definiría como:

un conjunto de conocimientos y capacidades que permiten el ejercicio de la actividad profesional conforme a las exigencias de la producción y el empleo. Cada cualificación se organiza en unidades de competencia. La unidad de competencia es el agregado mínimo de competencias profesionales, susceptible de reconocimiento y acreditación parcial. Cada unidad de competencia lleva asociado un módulo formativo y/o profesional, donde se describe la 


\section{tgphiyg $\mathbf{4 8}$}

formación necesaria para adquirir esa unidad de competencia. Un conjunto de unidades de competencia forman la cualificación profesional que son los referentes para elaborar después las ofertas formativas conducentes a títulos de Formación Profesional y/o certificados de profesionalidad (Ministerio de Educación y Formación Profesional, 2020).

Por tanto, podríamos decir que lo que se enseña y aprende en los, actualmente, más de 180 títulos de Formación Profesional impartidos en más de 3.000 centros públicos o privados sería aquello que el sistema productivo demanda o, por lo menos, aquello que los diferentes agentes productivos y educativos consideran necesario para que un trabajador eficiente se incorpore a un determinado puesto de trabajo. Es importante subrayar que no es el Ministerio de Educación y FP de manera arbitraria quien completa el catálogo, sino que éste es el encargado de recoger las diferentes necesidades a través de un consenso contrastado entre los operadores del sistema. Esto da lugar a un proceso complejo y largo de detección, definición, contraste, corrección y finalmente publicación en el Catálogo Nacional de las Cualificaciones Profesionales.

Es también importante indicar que los títulos de FP tanto de grado medio como de grado superior en España, desde la implantación de la LOGSE en 1990, son títulos específicos con unos currículos especializados donde sólo encontraremos módulos profesionales — asignaturas - ligados a unidades de competencias profesionales. Es decir, no hay materias comunes del tipo Matemáticas, Lengua Española o Historia, aunque sí se cuenta con módulos transversales como Inglés Técnico, Formación y Orientación Laboral o Empresa e Iniciativa Emprendedora. Se entendió entonces que las unidades de competencias generales ya estaban aprendidas en la enseñanza general, bien en la Educación Secundaria Obligatoria (ESO), bien en el Bachillerato correspondiente, y así se ha mantenido en las siguientes leyes orgánicas del Sistema Educativo y, también, en las orgánicas específicas de FP. Aunque esto, quizás, puede chocar con las recomendaciones hechas por el Consejo de Europa en 2018. Esto evidencia lo que ha seguido siendo una discusión relevante en el ámbito curricular sobre cómo los estudios de Formación Profesional tienen que contribuir a la adquisición de las competencias clave y transversales. En concreto las recomendaciones, entre otras, que hace el Consejo son:

\section{Contribuir al desarrollo de las competencias clave prestando especial atención a:}

2.1. Elevar el nivel de adquisición de las capacidades básicas (lectoescritura, cálculo y capacidades digitales básicas) y apoyar el desarrollo de la competencia para aprender a aprender como base mejorada constantemente para el aprendizaje y la participación en la sociedad desde la perspectiva de toda una vida.

2.3. Fomentar la adquisición de competencias en ciencia, tecnología, ingeniería y matemáticas (CTM), teniendo en cuenta su vínculo con las artes, la creatividad y la innovación y motivar a los jóvenes, en especial las chicas y las mujeres jóvenes, para que opten por carreras profesionales en estos ámbitos;

2.4. Ampliar y mejorar el nivel de competencias digitales en todas las fases de la educación y la formación, así como en todos los segmentos de la población.

2.6. Aumentar el nivel de las competencias lingüísticas en lenguas tanto en lenguas oficiales como en otras y animar al alumnado a que aprenda distintas lenguas relevantes para su situación laboral y vital y que puedan contribuir a la comunicación y la movilidad transfronterizas. (Consejo de la Unión Europea, 2018)

Quiero señalar también que ésta es una diferencia importante con respecto a la mayoría de los currículos de la Formación Profesional de los países europeos de nuestro entorno. En ellos, la FP lo es mayoritariamente de un nivel correspondiente a nuestro grado medio y entienden necesario seguir impartiendo estas asignaturas generales no específicas dentro de los currículos oficiales de la FP.

También es importante recordar una evidencia y es que el sistema de actualización de nuestros títulos, 


\section{tapbiya $\mathbf{4 8}$}

correspondientes a las necesidades formativas de nuestros sectores productivos, es lento. Desde que los agentes del sistema detectan una determinada necesidad hasta que esto cristaliza en una cualificación y finalmente en un módulo profesional 0 en otra unidad formativa, a veces pasan años, cuando no lustros. Éste, por ahora, es uno de los elementos conflictivos del sistema, pues las empresas y la sociedad demandan constantemente una actualización que parece que siempre llega tarde.

Finalmente, es necesario recordar que la Formación Profesional del Sistema Educativo se imparte en más de 3.000 centros educativos homologados. El porcentaje mayor por alumnado matriculado es el de los centros públicos, que pueden ser de oferta mixta donde se imparte ESO, Bachillerato y FP, o bien específicos, donde solo se imparte FP. En ambos casos, los centros son dependientes tanto a nivel curricular como a nivel de gestión de los recursos de las respectivas Comunidades Autónomas, que son quienes tienen la competencia real de la gestión de la Formación Profesional. También, existe un alto porcentaje de centros concertados y privados que gozan de una mayor autonomía de gestión de los recursos.

En los centros públicos el acceso, asignación a centros y organización del profesorado depende de las respectivas Comunidades Autónomas, cuyo sistema es el funcionarial. En los centros concertados y privados, el acceso, reclutamiento y gestión del profesorado depende de la empresa educativa correspondiente.

\section{Formación Audiovisual en la FP}

Hago esta introducción sobre el sistema educativo específico de Formación Profesional, por un lado, para que se pueda entender mejor dónde y cómo están encuadradas las enseñanzas audiovisuales dentro del marco general del sistema de FP. Pero también para dar a conocer el funcionamiento de estas etapas educativas que son las grandes desconocidas del sistema educativo del país. Quizás ese desconocimiento sea una de las razones por las que nuestra matrícula con respecto a los estudios académicos es desproporcionadamente baja en porcentaje con respecto a lo que ocurre en nuestro entorno europeo.

Por tanto, cuando queramos referirnos a estudios audiovisuales de Formación Profesional estaremos señalando a aquellos títulos que específicamente tienen cualificaciones profesionales relacionadas con los sectores productivos y de servicios de las industrias de la comunicación y del entretenimiento. Además, estos estudios se encuadran en las titulaciones de Grado Medio y Superior de la Familia Profesional de Imagen y Sonido u otras familias profesionales afines. También se enmarcan dentro de los Títulos Profesionales de Grado Superior de las Enseñanzas Artísticas de la Familia profesional de Comunicación Gráfica y Audiovisual. Estas enseñanzas tienen una estructura similar a las anteriores, pero se imparten en escuelas de arte en vez de institutos de Educación Secundaria y Formación Profesional.

Por otro lado, hay que reflexionar, también, sobre una hipotética y, quizás, necesaria competencia transversal en cultura audiovisual para otros títulos no relacionados con la familia profesional de Imagen y Sonido.

Este artículo quiere describir y señalar los elementos de conflicto o mejora de estos estudios audiovisuales de Formación Profesional, la demanda actual por parte de los estudiantes, la oferta de estos estudios por parte de los centros educativos y las salidas profesionales. Al mismo tiempo, quiero reflexionar sobre las metodologías, programas educativos y recursos utilizados en la FP de esta familia profesional, así como la idoneidad de los perfiles del profesorado y la participación de la empresa en la formación de los estudiantes. 


\section{Estudios específicos en audiovisuales en Formación Profesional y en Enseñanzas Artísticas Profesionales}

Las características comunes de acceso para los estudios profesionales y los estudios artísticos profesionales son las siguientes: para los títulos de Grado Medio hay que acreditar tener el título de ESO o equivalente; para los de grado superior hay que tener el título de Bachillerato o equivalente. Además, en el caso de las titulaciones de enseñanzas artísticas es requisito pasar una prueba específica de aptitud artística.

Todos los títulos tienen una duración de 2.000 horas. Dentro de ese montante de horas están incluidos un módulo de prácticas obligatorias en una empresa 0 institución del sector de casi 400 horas y un módulo de proyecto final.

Los ciclos están organizados habitualmente en dos cursos escolares completos. Desde el curso 2019/2020 también se cuenta con los denominados cursos oficiales de especialización, que tienen una duración variable. Las asignaturas en FP se denominan módulos y normalmente abordan una unidad de competencia, como se ha explicado anteriormente.

Dentro de las enseñanzas artísticas que tienen una parte sustancial de sus competencias ligadas al audiovisual se encuentran los títulos de Técnico Superior de Artes Plásticas y Diseño de la Familia de Comunicación Gráfica y Audiovisual:

- Técnico Superior en Animación.

- Técnico Superior en Cómic.

- Técnico Superior en Fotografía.

- Técnico Superior en Gráfica Audiovisual.

- Técnico Superior en Gráfica Interactiva.

- Técnico Superior en Gráfica Publicitaria.

Estos cursos tienen una oferta muy minoritaria, se imparten en mayor medida en las Escuelas de Arte —antiguas escuelas de oficios - y cuentan con un cuerpo de docentes específicos diferenciado, por sus especialidades y características de formación y acceso, del cuerpo de profesores de Secundaria y de Profesores Técnicos de FP.

Brevemente, podemos señalar como elemento destacable la orientación artística, práctica, experimental e investigativa del oficio. A pesar del atractivo que tienen, estas enseñanzas son las grandes desconocidas tanto para las empresas como para la sociedad en general. Desarrollan su práctica con metodologías mayoritariamente activas, trabajando en proyectos y retos investigadores y exploratorios, pese a que, en general, tienen una dotación muy baja en recursos materiales y humanos. Aunque cabe señalar que esta realidad es muy variable en función de la sensibilidad de cada una de las Comunidades Autónomas de las que dependen.

Asimismo, es significativo que, pese a la altísima proyección laboral que tienen estas titulaciones, se dé tan poca relevancia a su difusión dentro del sistema de orientación. Esto tiene como consecuencia el desconocimiento de estas enseñanzas para la mayoría de los estudiantes y familias. La escasa atención que prestan a estos títulos desde las administraciones públicas deriva en una invisibilidad llamativa cuando se explora el mapa de centros que ofertan estos estudios.

Esta falta de oferta y esta desatención en la orientación provocan que, ante el desconocimiento de las oportunidades de formación en este campo profesional, las vocaciones se despierten tardíamente. Así pues, dado que son profesiones que tienen un fuerte componente vocacional, las personas acaban buscando la formación en estas disciplinas en academias, cursos no reglados, formación no regulada o bien en centros privados no homologados que acogen un variopinto ecosistema de escuelas y currículos.

Desde mi punto de vista, considero necesario reforzar la oferta de aquellos títulos de enseñanzas artísticas que hoy 
tienen una fuerte demanda en el mercado laboral como, por ejemplo, las especialidades de Animación, Gráfica Interactiva y Gráfica Audiovisual.

Al mismo tiempo, se debería dotar a los centros con las instalaciones y con los medios técnicos necesarios. También, hay que reforzar el sistema de formación y actualización de este profesorado. Por otra parte, sería importante la revisión del sistema de acceso a la docencia de estas enseñanzas, mediante el aumento de la presencia de profesionales en activo como profesores especialistas.

En cuanto a las enseñanzas de FP del sector audiovisual, tenemos los títulos de Técnico y Técnico Superior de FP, pertenecientes a la Familia de Imagen y Sonido, pero que también se encuadran dentro de la de Artes, más en concreto en la de Imagen Personal.

Estas titulaciones son herederas de los títulos y centros de FP de la Ley General del 70, que durante años fue una vía denostada por ser el camino que tomaban los expulsados del sistema académico y a la que se accedía sin ningún tipo de titulación. A pesar de la reforma integral realizada en 1990 con la LOGSE y posteriormente con la Ley de las Cualificaciones de la FP del 2002, estas titulaciones arrastran aún un estereotipo que las sitúa en una opción de segunda clase.

Actualmente, encontramos formación audiovisual específica en los siguientes títulos, algunos de los cuales se enmarcan en la familia más genuina del sector, la Familia Profesional de Imagen y Sonido:

- Técnico Grado Medio de Videodj y Sonido.

- Técnico de Grado Superior en Sonido.

- Técnico de Grado Superior en lluminación y Captación de Imagen.

- Técnico de Grado Superior en Producción de Audiovisuales y Espectáculos.

- Técnico de Grado Superior en Realización de Audiovisuales y Espectáculos.

- Técnico de Grado Superior en Animaciones 3d y Videojuegos.

También hay titulaciones que tocan de manera importante parte de las competencias audiovisuales. Es el caso de los títulos que se encuadran en la Familia Profesional de Imagen Personal:

- Técnico Superior en Caracterización y Maquillaje.

0 también, en la Familia de Artes y Artesanías, cuya titulación, a pesar de su nombre, forma a profesionales para el Departamento de Dirección Artística tanto para audiovisuales como en espectáculos:

- Técnico Superior Artista Fallero y Construcción de Escenografías.

Las enseñanzas de audiovisuales de la FP, tal y como pasa en el resto títulos de Formación Profesional, tienen una vocación profesionalizadora clara e indiscutible. Están muy pegados a las necesidades técnicas de los procesos, los flujos de trabajo, así como al manejo aplicado de las herramientas habituales. Se atiende a una capacitación para la realidad inmediata, pero, también, se tiene como objetivo añadir una visión más amplia del sector profesional, para que sea más fácil la recualificación y la formación continua. Es una evidencia para todos los agentes de este sector la necesidad de una formación que permita la actualización constante en un medio que se transforma tecnológicamente cada cinco años.

Cada uno de los títulos reseñados más arriba abordan la formación en uno o varios perfiles profesionales concomitantes:

- Técnico Grado Medio de Videodj y Sonido: forma perfiles profesionales como ayudante de plató de TV, ayudante de sonido en diversos tipos de producción o técnico de animación musical y visuales.

- Técnico de Grado Superior en Sonido: estaría en relación a la formación de perfiles profesionales como 
técnico de sonido para televisión, cine, radio o directos, producción y grabación musical o diseñador de sonido para audiovisuales.

- Técnico de Grado Superior en lluminación y Captación de Imagen: se ocuparía de los profesionales como son: operador de cámara ENG y TV, fotógrafo, iluminador de cine, televisión y espectáculos.

- Técnico de Grado Superior en Producción de Audiovisuales y Espectáculos: este título forma ayudantes de producción en el ámbito del cine, radio, televisión y espectáculos.

- Técnico de Grado Superior en Realización de Audiovisuales y Espectáculos tendría como objetivo de formación los perfiles de ayudante de realización para cine, video y televisión, regiduría de teatro y operador de montaje, cine y postproducción y efectos digitales de audiovisuales.

- Técnico de Grado Superior en Animaciones 3d y Videojuegos: un título más orientador y menos profesionalizador, que abarca competencias diversas en el marco de la industria de la animación y los videojuegos, pero que pretende formar profesionales como modeladores 3D, animadores y técnicos de desarrollo de videojuegos.

- Técnico Superior en Caracterización y Maquillaje: encuadrado en una familia como es la de Imagen Personal, tendría como objetivos la formación de técnicos de maquillaje y caracterización de personajes para cine, televisión y espectáculos de artes vivas.

- Técnico Superior Artista Fallero y Construcción de Escenografías: finalmente, este título de Grado Superior tiene una denominación que parece encasillarlo en el arte del diseño y construcción de fallas. Sin embargo, tiene en su currículo, en más del 70\% de las competencias, como objetivo la formación para el diseño y ejecución de proyectos escenográficos para las industrias del cine, la televisión y los espectáculos de artes vivas. Sin embargo, por la concreción del nombre, solo está implantado en la Comunidad Valenciana. Este hecho, lamentablemente, obliga a los interesados en esta formación a acudir a programas variopintos en escuelas y academias no reguladas.

\section{Políticas públicas sobre la oferta de las enseñanzas de audiovisuales del sistema de FP e inserción laboral}

Las referidas enseñanzas se imparten en centros públicos y también en centros privados de toda España. Están concentrados, lógicamente, en ámbitos urbanos. Especialmente allí donde el tejido industrial audiovisual es más potente por número de empresas, peso en el mercado de trabajo o en el PIB. Es el caso de las áreas metropolitanas de Madrid y Barcelona y algunas de las capitales de diferentes Comunidades Autónomas.

La oferta es muy abundante en el ámbito privado — de pago — por no poder satisfacerse esta demanda con la oferta presencial de plazas desde el ámbito público.

En el caso de la Comunidad de Madrid, que podría ser un buen ejemplo de territorio con gran concentración de estas enseñanzas, según datos de la Dirección General de Bilingüismo y Calidad de la Enseñanzas de la Consejería de Educación y Juventud en el curso 2018-19 titularon 17.524 personas en Ciclos Formativos de Grado Superior. Dentro de esa cifra, 1.641, casi un 10\% del total de alumnos, fueron estudiantes de Ciclos de la Familia de Imagen y Sonido. Entre ellos, 697 titularon en Centros Públicos y 944 lo hicieron en Centros Privados.

Quiero subrayar aquí que esa proporción de FP de pago tan alta solo se da en Ciclos de altííma demanda por parte de los estudiantes como en el caso de la Familia Sanitaria.

\footnotetext{
${ }^{1}$ Ver Informe 2020 sobre el sistema educativo en la Comunidad de Madrid. Curso 2018-2019 (Consejo Escolar de la Comunidad de Madrid, 2020).
} 


\section{tapbiya $\mathbf{4 8}$}

Estos datos ponen el foco sobre las estrategias y acciones relativas a la oferta pública de la enseñanza postobligatoria que han de adoptar las políticas públicas en Formación Profesional o en la Educación en general.

Como hemos visto se produce tensión en la demanda del alumnado por diversos factores como la moda, permanente 0 pasajera, la relevancia y proyección social o el atractivo intrínseco de la disciplina. Todo ello tiene como consecuencia un número altísimo de solicitudes que no se pueden cubrir con la oferta existente, bien por su coste, bien porque las proyecciones o estudios sobre inserción laboral indican que estos egresados no podrán ser absorbidos por sectores que ya están saturados. Por contra, nos encontramos con la oferta de otros títulos de Formación Profesional con mejor perspectiva de empleo, pero que, sin embargo, son obviados y en muchos casos quedan vacantes.

Cabe destacar que el intento de redirección de la demanda hacia familias profesionales con más empleabilidad provoca que parte de estas solicitudes que no obtienen plaza deriven al sector privado. Estos centros privados lógicamente tienen como objetivo el negocio, pero su oferta, que forma y certifica estas mismas competencias profesionales, termina por agravar la tensión entre personas formadas en audiovisuales y el empleo real en las empresas.

Como consecuencia del alto número de egresados que suman los centros públicos y privados, podemos concretar, a su vez, al menos cuatro consecuencias negativas. Todas ellas bien conocidas en los centros de formación audiovisuales tanto de Formación Profesional como de grados universitarios y que tan bien ha descrito Remedios Zafra en El entusiasmo. Precariedad y trabajo creativo en la era digital (Zafra, 2017):

- La bajada de los salarios de los profesionales de estos sectores "atractivos y vocacionales" y, por tanto, la precarización de este sector desde el punto de vista laboral, pues las empresas tienen a su disposición una bolsa enorme de profesionales bien formados con expectativas de ocupar un puesto de trabajo.

- La "amateurización" de estos profesionales que desarrollan su trabajo en la forma de colaboraciones con las empresas, transitando con figuras de falsas becas, meritoriajes o subcontratación parcial como falsos autónomos.

- La "sobrecualificación" para determinados puestos técnicos donde compiten titulados de FP de Grado Medio y Superior con graduados universitarios y titulados en diversos másteres universitarios que no encuentran inserción en la industria de su propio nivel de titulación y que buscan más abajo la inserción. A estos perfiles hay que sumar el de las personas formadas en toda una pléyade de cursos no homologados por el Sistema Educativo General.

- La mala fama o consideración social de estos estudios por ser poco acordes con las necesidades del mercado laboral y productivo. En muchas ocasiones se llegan a considerar, más que como una profesión, como un hobby.

Sin embargo, algunos datos contradicen estas evidencias tan claras para los tutores de los centros de FP o para los coordinadores de prácticas universitarias. Así, si tomamos otra vez como ejemplo a la Comunidad de Madrid y observamos el grado de inserción de las personas tituladas de la Familia Profesional de Imagen y Sonido, los datos indican que esta inserción supera el 75\% del total de egresados. También el estudio muestra que la inserción laboral general en todas las familias de FP de estas titulaciones es de 75,4\%, aunque dicho estudio no puede precisar si están trabajando en el sector productivo audiovisual o de cuánto tiempo es el contrato de trabajo (ver Consejo Escolar de la Comunidad de Madrid, 2020).

\section{Las prácticas en las empresas como elemento clave para la FP}

Entre los elementos destacados de este tipo de estudios resalta la colaboración formativa de las empresas, que se expresa en el módulo obligatorio de Formación en Centros de Trabajo, FCT, que se desarrolla durante el último 


\section{taphiya $\mathbf{4 8}$}

trimestre del segundo año. Es un módulo obligatorio de 380 horas que implica la participación como tutores, mentores y profesores de los empleados y profesionales especializados de las empresas del sector audiovisual y de espectáculos. Esta parte de la formación es clave para perfilar la formación final, pero, también, es un importante elemento de mejora de los programas y la práctica docente de los centros. Este módulo facilita la retroalimentación mediante el flujo de documentos de programación formativa, documentos de evaluación, entrevistas e información no formal.

En este punto es importante señalar que estas prácticas están fuera del ámbito normativo laboral. No implican dar de alta en la Seguridad Social al estudiante y no son remuneradas, aunque pueda a veces éste recibir alguna compensación para transporte 0 manutención dependiendo de la empresa 0 de la Comunidad Autónoma. Estas prácticas en centros de trabajo tampoco están reconocidas en el contrato de los empleados encargados de realizar el trabajo de tutorización o de instrucción. Esta doble gratuidad choca con el rendimiento real que supone para las empresas que los acogen, ya que aumenta la mano de obra en el lugar de trabajo y es un elemento que cíclicamente aparece como conflictivo para sindicatos, asociaciones de empresarios o colectivos de estudiantes.

Sin ir más lejos, la intención del actual gobierno de que fuese obligatoria la inscripción en la Seguridad Social de los estudiantes en prácticas en una empresa y que esta inscripción y su coste fuese asumido por la empresa en cuestión provocó un enorme revuelo y la marcha atrás de la medida anunciada².

\section{Metodologías y Profesorado}

Estas enseñanzas se imparten mayoritariamente evitando las pedagogías academicistas más conceptuales y las metodologías conductistas apoyadas en la repetición de rutinas para asentar el aprendizaje. La propia naturaleza de las técnicas y procesos de producción en los entornos audiovisuales y del espectáculo hace que en la mayoría de los casos los programas formativos intenten ensayar modelos cercanos a las metodologías activas, mediante el trabajo por retos 0 por proyectos. De esta forma, se integran diversos ciclos en un único proyecto o producto. Los talleres y laboratorios son las localizaciones de rodaje, los estudios de grabación, aulas escénicas o platos de tv, entre otros.

Cuando se analizan los reales decretos que establecen los títulos, la propia organización de los módulos ya conlleva, implícitamente, que la organización, a su vez de los aprendizajes, se escore hacia los proyectos en grupos mediante prácticas reales.

Por otro lado, es necesario señalar como carencia general de los actuales títulos de FP de las enseñanzas audiovisuales la falta de módulos o contenido que contextualicen el ciclo en el área de la comunicación audiovisual y en la propia historia de los medios audiovisuales. Esto ocurre en las titulaciones de la LOE (no sucedía con los títulos de la LOGSE). Solamente en el ciclo de Sonido hay un módulo denominado Comunicación y expresión sonora. En el resto de títulos de la familia no hay ningún módulo que aborde estas cuestiones que permiten a los estudiantes tener herramientas de compresión de lo audiovisual desde una perspectiva crítica, sistémica y evolutiva. Esta carencia contrasta y compromete el objetivo expresado en toda la Formación Profesional de añadir competencias que permitan una visión más amplia y crítica de la profesión. Faltan contenidos que faciliten elementos al alumnado para situarse contextualmente con el fin de seguir formándose a lo largo de la vida.

Respecto a los docentes encargados de la enseñanza de estos Ciclos Formativos, como en el resto de la FP, estaríamos hablando de dos cuerpos docentes de funcionarios para la impartición de la docencia, en este caso, en el sector público. Estos docentes serían en un 50\% del cuerpo de profesorado de secundaria. Dentro de dicho cuerpo están los

\footnotetext{
${ }^{2}$ Ver "El Gobierno obligará también a cotizar por los 725.000 estudiantes de FP que hacen prácticas" (eldiario.es, 2019).
} 
docentes de la especialidad de Procesos y Medios y de Comunicación, los de la especialidad de inglés que imparten los Módulos de Inglés Técnico y los que imparten Formación y Orientación Laboral y Empresa e Iniciativa Emprendedora que serían de la especialidad de Formación y Orientación Laboral. Por otro lado, otro 50\% de los docentes serán los pertenecientes al cuerpo de Profesores Técnicos de FP de la especialidad de Imagen y Sonido. En ambos casos los requisitos de acceso son similares: graduados universitarios, mayoritariamente procedentes de las enseñanzas de Comunicación Audiovisual y Periodismo, con un acceso mediante oposición a los dos cuerpos y con una carrera profesional que tiene como principal mérito la antigüedad y sin prácticamente evaluación, excepto los exámenes de oposición y la evaluación inicial como funcionario en prácticas el primer año.

Esta descripción ya nos anticipa los problemas principales que nos encontramos en la docencia de la FP y que serían: la ausencia de una carrera profesional propiamente dicha, la carencia de reconocimiento para aquellos docentes que previamente hayan desarrollado experiencia profesional en la industria y la ausencia de evaluación para cualquier tipo de mérito profesional del sector.

El principal reto que se les va a demandar a estos docentes, tanto a los del cuerpo de secundaria como a los del cuerpo de profesores técnicos, es poseer un conocimiento aplicado y específico de técnicas, tecnologías, procesos y flujos de trabajo que son altamente especializados y diferentes entre sí y que, por tanto, requerirán de un altísimo grado de especialización y actualización.

Para hacernos una idea, podríamos poner de ejemplo los perfiles docentes necesarios en un centro donde se imparte la oferta al completo de la Familia Profesional de Imagen y Sonido. En este caso el profesorado de estas especialidades tendría que tener competencias profesionales en perfiles tan distintos como iluminación de espectáculos, electroacústica, desarrollo de videojuegos, sesiones de música para djs, realización de cine y video o fotografía publicitaria.

Una de las demandas más habituales entre el profesorado, lógicamente, es la de crear más especialidades en cada familia profesional para que el factor especialización fuese abordable de una manera razonable. Pero no es la única, pues también se demanda un aumento de la formación específica dentro de las jornadas de trabajo (no como una carga añadida a las largas jornadas lectivas), la realización de estancias formativas en empresas durante periodos largos que permitiesen la actualización profesional y la puesta al día con la realidad productiva donde luego se insertarán sus estudiantes.

Por otro lado, los centros de Formación Profesional reclaman, también, la inclusión, de manera sistemática, de profesorado especialista procedente del mundo profesional, similar al de un Profesor Asociado de Universidad, para ayudar como apoyo, especialmente durante las horas lectivas prácticas.

Mientras se ordena de otra manera la gestión de los docentes, la mayoría de ellos, cuando tienen estabilidad, es decir, cuando ya han aprobado las oposiciones y tienen destino definitivo, dedican una gran parte de su tiempo no lectivo a la actualización de conocimiento, perfeccionando su manejo de las nuevas herramientas y procedimientos. El rápido cambio de las tecnologías y de los flujos de trabajo en las empresas provoca que la obsolescencia del conocimiento sea vertiginosa.

Después de ejercer la función de director en un centro de formación audiovisual durante más de veinte años, he podido observar la trasformación radical de las profesiones del sector audiovisual y del espectáculo. Por ello, desde mi punto de vista, es muy meritorio el nivel medio de cualificación de los profesores de FP de estas especialidades, dadas las condiciones adversas más arriba explicadas. Sin ninguna duda, la actualización ha sido, de lejos, el principal reto y preocupación tanto para los profesores como para los centros.

En todo caso, esta problemática sobre la actualización, tan necesaria para estas enseñanzas, no quita tampoco la 
necesidad de una profunda revisión del modelo funcionarial, tal y como está concebido actualmente.

Otro de los retos que nos encontramos en los centros de Formación Profesional es la necesidad de inversión para actualizar los recursos técnicos, básicos para una enseñanza de este tipo basada en el aprendizaje de los procesos con herramientas, etc. Así lo han remarcado repetidamente los centros y así consta en los documentos generados por la Asociación de Centros de Formación Profesional FPEmpresa ${ }^{3}$, entidad de la que actualmente soy presidente. En dichos documentos anuales se reitera la necesidad de ampliar la inversión para la actualización tecnológica y para la mejora en la formación de los docentes.

\section{Competencia Comunicación y Cultura Audiovisual}

Finalmente, quisiera señalar la importancia de contemplar una competencia centrada en la comunicación y la cultura audiovisual dentro de otros títulos de FP. Dicha competencia podría insertarse en el módulo transversal de Empresa e Iniciativa Emprendedora, como señala en su página web uno de los centros pioneros en emprendimiento, me refiero al Centro Valnalón del Principado de Asturias:

El objetivo general del módulo profesional Empresa e Iniciativa Emprendedora es que el alumnado de formación profesional sea capaz de desarrollar la propia iniciativa emprendedora tanto en el ámbito empresarial y hacia el autoempleo como la actitud intraemprendedora hacia la asunción de responsabilidades y funciones en el empleo por cuenta ajena. Este objetivo se concreta en los siguientes específicos, (...) reconocer y aplicar las competencias laborales de comunicación, liderazgo y motivación, valorando su importancia para lograr un eficaz funcionamiento de las organizaciones, así como la mejora del ambiente de trabajo y el aumento de la implicación y el compromiso de las personas que forman parte de ella. (Valnalón, 2020)

Este objetivo común a todos los ciclos de FP necesitaría profundizar en elementos como son la estructura de los medios de comunicación, storytelling, narrativas audiovisuales clásica y emergentes, redes sociales, características de la comunicación y el lenguaje audiovisual, etc. De hecho, ya se están desarrollando programas formativos en este sentido que, sin embargo, carecen de las suficientes horas lectivas necesarias para la implementación de proyectos de emprendimiento que incluyan en su estrategia de creación la comunicación audiovisual.

\section{Conclusión}

Las enseñanzas de audiovisuales de Formación Profesional se encuentran enmarcadas en el ámbito de la FP del sistema educativo. Este ámbito en los últimos años se está posicionando mejor dentro del sistema educativo general. Como consecuencia se observa un aumento del número de estudiantes matriculados. Estamos acercándonos a los números que hemos de alcanzar si miramos a la mayoría de los países de nuestro entorno. Venimos de una situación en la que la FP estaba poco valorada socialmente. Era una alternativa educativa secundaria para la mayoría de la sociedad de nuestro país. Ahora esa visión está cambiando. Las familias y empresas miran de otra manera a estas enseñanzas.

También, cabe destacar que la Formación Profesional del sector audiovisual tiene una estructura curricular, como el

\footnotetext{
${ }^{3}$ FPEmpresa es una asociación sin ánimo de lucro que reúne a Centros de Formación Profesional públicos y concertados de toda España. Desde 2012, trabaja con la finalidad de impulsar y fomentar la Formación Profesional a través del intercambio de experiencias y buenas prácticas entre los centros que componen la asociación. Página web: https://fpempresa.net/
} 


\section{taphiya $\mathbf{4 8}$}

resto de la FP, basada en el Sistema Nacional de Cualificación Profesionales, muy ligada a la realidad productiva, aunque la lentitud del sistema haga que a veces se produzca un retraso entre la realidad productiva y el contenido de los currículos.

Por otro lado, hemos señalado cómo las enseñanzas artísticas son una modalidad arrinconada que, sin embargo, podría tener más importancia. Sus títulos son demandados, pero están en una esquina poco visible tanto para el sistema de orientación como para la sociedad en general. No obstante, el fuerte componente vocacional hace que los adultos busquen realizar formación no reglada en este campo para satisfacer su vocación.

De la misma forma, las enseñanzas de FP de las familias relacionadas con lo audiovisual tienen un atractivo que las hace ser muy demandadas. Las solicitudes están por encima de la oferta educativa de las administraciones públicas. Estas intentan intervenir frenándola, pero generan como consecuencia que estas vocaciones por las profesiones audiovisuales se dirijan hacia la oferta privada de estudios profesionales.

La FP de enseñanzas audiovisuales es ambiciosa con respecto al espectro que abarca. Sin embargo, la estructura actual en cuanto a organización de los centros y en cuanto al sistema de reclutamiento y gestión de los docentes hace que se produzca una tensión nacida del intento de dar respuesta a las necesidades del sector que está en un constante y vertiginoso cambio.

Se observa, por otro lado, la preponderancia de metodologías activas y prácticas. Esto hace que, en consecuencia, el proceso de aprendizaje sea de más calidad que si se usasen didácticas pasivas o basadas solamente en los conocimientos acumulados, como ocurre en los programas universitarios.

Es importante, también, señalar que la estancia del alumnado en las empresas a través del módulo obligatorio de Formación en Centros de Trabajo produce una conexión rica y más dosis de realismo a estas enseñanzas.

Por último, cabe destacar que es necesario una importante inversión para la actualización constante de los equipos y materiales, si se quiere seguir la estela del propio sector. También es necesaria una reflexión sobre la utilidad de ampliar la competencia en cultura audiovisual a otros títulos de FP, para ayudar a desarrollar las competencias ligadas a la iniciativa emprendedora de estos titulados. 


\section{taphiya $\mathbf{4 8}$}

\section{Bibliografía}

BOE (1970). Ley 14/1970, de 4 de agosto, General de Educación y Financiamiento de la Reforma Educativa.

https://www.boe.es/buscar/doc.php?id=BOE-A-1970-852

BOE (1990). Ley Orgánica 1/1990, de 3 de octubre, de Ordenación General del Sistema Educativo (LOGSE).

https://www.boe.es/eli/es/lo/1990/10/03/1

BOE (2002). Ley Orgánica 5/2002, de 19 de junio, de las Cualificaciones y de la Formación Profesional.

https://www.boe.es/eli/es/lo/2002/06/19/5/con

BOE (2006). Ley Orgánica 2/2006, de 3 de mayo, de Educación (LOE).

https://www.boe.es/diario_boe/txt.php?id=BOE-A-2006-7899

CONSEjO de la Unión EUROPEA (2018). Recomendación del Consejo, de 22 de mayo de 2018, relativa a las competencias clave para el aprendizaje permanente (Texto pertinente a efectos del EEE) (2018/C 189/01). Diario Oficial de la Unión Europea.

https://eur-lex.europa.eu/legal-content/ES/TXT/PDF/?uri=CELEX:32018H0604(01)\&from=SV

CONSEjo Escolar de LA COMUNIDAD DE MAdRID (2020). Informe 2020 sobre el sistema educativo en la Comunidad de Madrid. Curso 2018-2019. Madrid.

https://www.comunidad.madrid/publicacion/1354812215401

ELDIARIO.ES (2019). El Gobierno obligará también a cotizar por los 725.000 estudiantes de FP que hacen prácticas. eldiario.es, 12 de enero de 2019.

https://www.eldiario.es/sociedad/cotizacion-seguridad-social-fp_1_1757416.html

Ministerio de Educación y FORMación PROFESIONAL (2020). Quées una competencia profesional. En Todofp.es.

https://www.todofp.es/acreditacion-de-competencias/informacion-general/que-es-competencia-profesional.html

SNCFP (2020). Catálogo Nacional de Cualificaciones Profesionales. Ministerio de Educación y Formación Profesional.

http://incual.mecd.es/documents/35348/80300/CNCP_listadoQ.pdf/

Valnalón, Centro Valnalón del Principado de Asturias (2020). Objetivos.

http://www.valnaloneduca.com/eie/cont/objetivos

ZafRA, R. (2017). El entusiasmo. Precariedad y trabajo creativo en la era digital. Barcelona: Anagrama. 


\title{
Resumen.
}

Partiendo de una breve descripción del Sistema de Formación Profesional en España y de los elementos clave de su funcionamiento, como son las cualificaciones profesionales y los títulos de FP, se describirá posteriormente cuáles son aquellos estudios específicos que tienen relación directa con la formación audiovisual y sectores profesionales cercanos. El siguiente paso será describir cuáles son y cómo son los objetivos formativos de las enseñanzas audiovisuales en Formación Profesional. Asimismo, se señalarán tanto los elementos característicos como las claves, conflictos o puntos de mejora de estos estudios audiovisuales de Formación Profesional. Algunos ejemplos de dichos elementos son la demanda actual por parte de los estudiantes, la oferta de estos estudios en los centros educativos y las salidas profesionales. Además, el artículo contiene una reflexión sobre las metodologías, pedagogías y recursos utilizados en la FP de esta familia profesional, la idoneidad de los perfiles del profesorado y la participación de la empresa en la formación de los estudiantes. Al final se abre una especulación de si hay o no una necesidad en otros títulos de FP no específicos de los sectores de la comunicación audiovisual de introducir como competencia transversal la cultura audiovisual.

Palabras clave. Formación profesional; Competencias curriculares; Industrias de la comunicación; Sistema educativo; Formación de profesores;Metodologías activas; Orientación profesional.

\begin{abstract}
.
Starting from a short description of the Vocational Education and Training (VET) System in Spain and from the key elements of its functioning, that is, professional qualifications and VET degrees, we will explain which specific studies are directly linked to audio-visual training and other related sectors. The next step will be to describe the training objectives of the audio-visual teachings in VET. Furthermore, we will analyse the main aspects, the keys, the conflicts, and the improvement points of these audio-visual studies in VET. Some examples of the afore-mentioned aspects are: the current demand by students, the offer of these studies in VET centres and the career opportunities. In addition to all this, this article contains a reflection on methodologies, teaching abilities, resources used in VET within this professional branch, the adequacy of teachers' profile and the participation of companies in students' training. At the end, there will be a general discussion on whether it is necessary to include audio-visual culture as a cross-curricular competence in other VET degrees that are not particularly related to audio-visual communication sectors.
\end{abstract}

Key-words. Vocational education and training; Curricular competencies; Communication industries; Education system; Teacher training; Active methodologies; Professional orientation.

Luis García Domínguez

Profesor de Secundaria de la Especialidad de Procesos y Medios de Comunicación Profesor Asociado del Departamento de Periodismo y Comunicación Universidad Carlos III de Madrid

Director del IES Puerta Bonita de Madrid

Presidente de la Asociación de Centros de Formación Profesional - FPEmpresa

Igarcia@iespuertabonita.com 